$\mathrm{pO}_{2}$ decreased in 10 of 11 dogs, and cortical $\mathrm{pCO}_{2}$ showed slight and transient decrease in the same experimental period accompanied with reduced regional cerebral blood flow.

In EEG, slight voltage depression and occasionally slow waves were observed in early stage after the infusion and these alterations were followed by gradual improvements.

Group 2: Hypervolemia seemed to be a factor of raising systemic blood pressure slightly. In this group, neither irregular respiration nor the alterations of cortical $\mathrm{pO}_{2}$ was observed. Cerebral blood flow increased, while cortical $\mathrm{pCO}_{2}$ showed gradual decrease. Intracranial pressure lowered in all cases. EEG revealed slight improvement.

Group 3: Cerebral blood flow increased slightly but the other parameters showed no remarkable alterations.

ifference of the effects of urea and mannitol on cerebral edema was emphasized and the effects of low molecular dextran on cerebral blood flow was noted in this report. From our expriments, the administration of urea seemed to remain some problems for its clinical use.

\title{
54. The Clinical Study on Steroid Therapy in Neurosurgery
}

\author{
Yoshikazu SAITo \\ 2nd Department of Surgery, Tottori University School of Medicine
}

The treatment with adrenocortical steroid hormone for cerbral edema has been commonly done with effect in acute cerebral injury, brain tumor operations and cerebral apoplectic strokes etc. But the dosage of the medicament is not always determined accurately and side effects of them are considered to be not so rare.

Clinical results in the present study on these problems as above described are as follows:

1) Postoperative rise in body temperature in patients with brain tumors was measured compared in two groups. In one group of 15 cases of brain tumors with out steroid therapy, the rise in body temperature after operation was remarkable. But the rise in body temperature in the other group of 50 cases of brain tumors after operation was controlled moderately well by administration of steroid hormone. The differnce between these two groups was very remakable and optimate dosage of steroid hormone was considered to exist between 1.5 and $2.0 \mathrm{mg}$. per $\mathrm{kg}$. of prednisolone.

2) The same tendency was found also in pneumo-and oleo-ventriculography. 
3) But in some cases the dosage of medicament was supposed to be too little and febrile states in high grade were experienced after steroid treatment which was finished too early.

4) In some another cases several side-effects were observed after the steroid treatment such as moon face, euphoric psychic state and bleeding from gastro-intestinal tracts etc.

Administration of prednisolone over $1000 \mathrm{mg}$ was made in some patients with metastatic brain tumors from lung or kidney with effect in the meaning of chemical adrenalectomy.

\title{
55. The Effect of Dexamethasone on Cerebrospinal Fluid Produciton Rate in the Dog
}

\author{
Yoshio Hashimoto, Osamu Sato, and Ryuichi Tsugane \\ 1st Dept. of Surgery, Nagoya University School of Medicine
}

The high mortality associated with brain swelling led very early to attempts to discover methods for its relief. More recently, adrenal cortical steroids have been found to be effective in controlling symptoms due to cerebral edema. However, few studies comparable to the above have been carried out to show the effectiveness of the corticoids or to help elucidate the mechanism of action.

In a previous paper, E.A. Bering, Jr. and one of us (O.S.) was able to show that c.s.f. production rate of normal dogs weighing between 15 and 20 kilograms was $0.048-0.031 \times 10^{-3}$ (CSFP-SSVP) $\mathrm{ml}$. per min. The same technique was used in present report applying ventricular perfusion method. I.V. Dexamethasone in the dose of $0.15 \mathrm{mgm}$. per kilogram body weight reduces the production rate down to nearly $50 \%$ of the rormal. The regression line can be indicated as follows: $0.024-0.042 \times 10^{-3}$ (CSFP-SSVP) ml. per min.

Diminution in the amount of c.s.f. production becomes apparent in $20 \mathrm{~min}$. after I.V. administration of Dexamethasone and reaches $-50 \%$ level in $30 \mathrm{~min}$. Also noticed was that I.V. Dexamethasone ( $0.15 \mathrm{mgm}$ per kilogram body weight) did alter cerebrospinal fluid (CSFP) or superior sagittal sinus pressure (SSVP).

It is beyond the scope of this paper to unveil the mechanism of the effectiveness of corticosteroid on cerebral edema, but it might be stated corticosteroids work closely on water-ion transport system. 